\title{
HUMANITIES
}

\section{THE LONG PATH OF ISLAM TO "THE BLACK COUNTRIES"}

\author{
Andrzej Krynski \\ Prof., PhD, Polonia University in Czestochowa, \\ e-mail : a.krynski@pnap.ap.edu.pl,orcid.org/0000-0001-9635-023X, Poland \\ Yves Merlin Kengne \\ MA, Institut Universitaire de Saint Jean Paul II de Bafang, \\ e-mail: kengneyvesmerlin@yahoo.fr, orcid.org/0000-0002-0119-6434, Cameroun
}

\section{Hameni Blaise}

MA, Institut Universitaire de Saint Jean Paul II de Bafang, e-mail : bhameni@yahoo.fr, orcid.org/0000-0002-9922-7947, Cameroun

\begin{abstract}
Islam as a mode of belief of the Arab peoples existed for several centuries in its original reign of the Near East. But as soon as it had a vigorous expansion, it turned to the "black countries" on the paths of Africa, first North, then South. In its desire to extend to the whole Africa, the conquering march of Islam was not smooth, it experienced many obstacles and wars. The islamization of the world took place because of many wars, but those wars did not always end with the victory of the Muslims, they sometimes led to the decline of the project of Islamization of the whole world.
\end{abstract}

Keywords: Islam, Muslim, black countries, Christian, expansion, war, conquest, islamization.

DOI: http://dx.doi.org/10.23856/2511

\section{Introduction}

Faire un article sur « LA LONGUE MARCHE DE L'ISLAM VERS LE «PAYS DES NOIRS » » revient à s'étendre sur l'origine de l'Islam, son fondateur, ses jeux et enjeux, ses objetifs et le long processus qui a conduit a son intriduction dans plusieurs région du monde, au dela de son leiu de création. Dans cet article il est question du processus $d$ 'islamisation $d u$ monde en passant par le continent africain plus précisément. Il est aussi question des jeux et enjeux des querres qui ont permis d'introduire l'islam dans plusieurs pays africains, et les obstacles qui ont jonchés le chemin de cette islamisation.

\subsection{L'islam sur le chemin de la conquête}

L'islam est né dans l'ouest de l'Arabie centrale au début du VIIe siècle.A peine dix ans après la mort du fondateur (en 632), les Arabes musulmans ont déjà conquis les cités les plus prestigieuses du Proche-Orient: Damas (en 636), Jérusalem(en 638), Alexandrie(en 642); et le mouvement foudroyant d'expansion n'était qu'à ses débuts.

Dès ses origines, l'islam se présenta ainsi comme la religion du succès, de la victoire par les armes. Cette étiquette lui est restée attachée à travers les siècles; une étiquette dont il faut vérifier le bien-fondéet les composantes en ce qui concerne la 
progression de l'islam en Afrique, au sud du Sahara, au cours d'un millénaire. Est-ce le succès des armes qui a fait qu'aujourd'hui près du tiers des Africains au sud du Sahara se disent musulmans?

\subsubsection{En Arabie: un peuple et un message}

L'Arabie, toute désertique qu'elle soit dans sa majeure partie, a toujours connu un peuplement en mouvement: des guerriers nomades et des caravaniers commerçants. Les seuls établissements permanents étaientles haltes d'étapes pour les caravanes. La Mecque en était une avec une population flottante selon les saisons, et sans grand enracinement. Il y avait d'autre part les campements nomades qui se déplaçaient au gré des pâturages et des occasions de razzia.

Le message de la foi au Dieu Unique, transmis par Mohammed dans une langue arabe claire et percutante, y tomba à un moment particulièrement mouvementé. Les fortes colonies juives, la présence de quelques chrétiens, judéo-chrétiens et nestoriens notamment, avaient mis bien des esprits en éveil. Par la force inspiratrice du langage et par la simplicité du dogme, le message nouveau donnait une réponse claire à beaucoup d'interrogations religieuses et servait en même temps de point de relliement pour un peuple en manque de cohesion, en recherche d'identité puisque, jusque là, il avait vécu en marge de l'histoire.

\subsubsection{Le message nouveau dans une chrétienté déchirée}

Pour sa part, le monde méditerranéen chrétienren dit la tâche facil eaux Arabes musulmans. Le césaropapisme de Bysance qui se croyait investide la mission de défendre par la force l'orthodoxie de la foi chrétienne avait été de tout temps mal supporté aussi bien par les nestoriens du Moyen Orient que par les Coptes monophysites de l'Egypte. Même les Eglises «orthodoxes» de tradition apostolique-Antioche, Jerusalem, Damas...-admettaient mal la main-mise, par empereur interposé, du patriarche de Constantinople. Ainsi, les conquérantsarabes furent accueillis un peu par-tout plutôt en libérateurs qu'en envahisseurs. Ceci d'autant plus, que la nouvelle religion, ressentie par beaucoup-même par des esprits aussi éclairés qu'un Saint Jean Damascène comme une secte chrétienne de plus, faisait montre d'un grand respect, d'une grande tolérance vis-à-vis du christianisme et des chrétiens. Il suffisait de reconnaître l'autorité musulmane et d'accepter sa «protection» moyennant un impôt spécial bien moins lourd que ce qu'avait exigé l'empereur de Bysance-pour pouvoir continuer à vivre sa foi chrétienne dans la paix et la sécurité.

\subsubsection{Le modèle fondateur de la cohabitation}

Dès le début, la cohabitation entre chrétiens et musulmans a donc connu un modèle pacifique certes, mais un modèle ambigû qui sera cause de. Tant d'incompréhensions, de suspicion et de conflits tout au long de l'histoire et jusqu'ànosjours: le musulman est convaincu qu'il respecte complètement la foi chrétienne en assurant au chrétien une place dans la cité musulmane et qu'il lui fait une faveur en le «protégeant».Le chrétien, lui, se sent dégradé en citoyen de second ordre, exclu des instances de décision, limité dans ses mouvements et ses droits. De fait, au 
cours des siècles, beaucoup de chrétiens n'ont pas voulu payer le prix fort du statut de «protégés» (dhimma) pour pouvoir pratiquer leur religion et ont tout simplement rejoint la communauté musulmane.

\subsubsection{L'islam: religion et Etat}

L'unité nouvellement acquise et fondatrice d'une communauté fraternelle basée sur un message simple mais dynamisant, Jança tout naturellement les Arabes sur le chemin de la conquête. Celle-ci allait dès le début dans deux directions opposées: vers l'est àtravers la Mésopotamie et l'Iran jusqu'au bord de l'Indus, dans le Pakistan actuel; vers l'ouest à travers l'Afrique du Nord pour atteindre l'Espagne moins d'un siècle après la mort de Mohammed.

Toutes ces conquêtes foudroyantesnefaisaient queconfirmer les musulmans dans une conviction qui avait ses racines dans la première communauté constituée par Mohammed à Médine: L'islam est à la fois religion et Etat, soumission au Dieu Unique à travers des rites clairement codifiés et, en même temps, modèle d'organisation de la société. Les deux sont révélés par Dieu. L'idéal religieux ne peut se réaliser pleinement qu'à travers l'idéal politique: la cité musulmane. Le premier but de l'Etat musulman est d'assurer aux musulmans toutes les chances d'atteindre l'ideal religieux, c'est-à-dire de garantir un ordre public bâti sur la loi islamique telleque révélée par le Coran. Même si, par la suite, l'unité effective de la communauté musulmane fut assez vite rompue, le principe même de l'islam, comme ordre politique, régi par le texte révélé, n'a jamais été mis en cause.

\subsection{Les chemins vers le bilâdas-sûdân}

Dès la conquête de l'Egypte, l'avancée musulmane cherchait aussi un chemin vers le bilâdas-sûdân, le pays des Noirs. Mais là, la pénétration devrait se faire bien plus modestement et sur un tout autre rythme, que celle du Moyen Orient ou celle de l'Afrique du Nord.

On peut distinguer principalement deux grandes voies de penetration de l'islam en Afrique au sud du Sahara:

-la voie maritime le long de la côte orientale,

-la voie des caravanes à travers le désert.

\subsection{LavalléeduNil}

Curieusement, lavoied'accèsversl'intérieurducontinentafricainqui semble la plus facile, une fois 1'Egypte conquise, celle par la vallée du Nil, restafermée à l'Islam pendant de longs siècles. L'islam du_t y contourner l'obstacle d'une forte présence chrétienne avant de pouvoir finalement le surmonter.

\subsubsection{La résistance des trois royaumes chrétiens de Nubie}

C'est le long du Nil, au Sud de la première cataracte et jusqu'au-delà du confluent du Nil blanc et du Nil bleu (Khartoum), que s'étendaient les trois royaumes chrétiens de Nubie: Nobatia, Makurria et Alwa. L'année même de la prise 
d'Alexandrie, une expédition arabe se dirigea contre Dongola, lacapitale de Makurria. La résistance des Nubiens chrétiens fut farouche et les Arabes durent se contenter d'un traité imposant aux Nubiens un tribut en esclaves, mais leur garantissant leur autonomie et le libre exercice de leur religion chrétienne (traité de Bakt en 651).

Par la suite, les essais ne manquèrent pas pour étendre l'influence de l'islam audelà d'Assouan sur la première cataracte, mais sans grand succès. Ce n'est que six siècles plus tard que la résistance nubienne s'effrita petit à petit. Politiquement de plus en plus divisée, là Nubie se trouvait alors complètement coupée du reste du monde chrétien.Vers la fin du XIIe siècle, avec l'arrivée de Saladin en Egypte, les liens avec le Patriarche d'Alexandrie se rompirent totalement, alors qu'il était le garant et le chef dela hiérarchie copte en Nubie. Les nombreux monastères, jusqu'alors foyers ardents de vie et de convictions chrétiennes et peuplés principalement de moines égyptiens, périclitèrent. L'Eglise de Nubies'éteignit peu à peu par- ce qu'elle manquait d'évêques et deprêtres.

\subsubsection{La chute et l'arabisation}

La prise de Dongolaen 1317 marqua le début de l'islamisation de la vallée duNil. Deux siècles plus tard (en 1505), Alwa, le dernier des trois royaumes, proche du hautplateau éthiopien,tomba à son tour. L'Ethiopie chrétienne, elle-même menacée par la pression musulmane, ne dut sa sur- vie qu'à l'intervention d'une expédition portugaise en 1543.

La chute des royaumes chrétiens de Nubie coïncida avec une nouvelle vague d'invasions de tribus arabes couvrant l'Afrique, d'est en ouest. Une infiltration de plus en plus massive d'éléments arabes, non seulement accélera l'slamisation, mais fut à l'origine de l'arabisation complète de ces religions.

Par la suite, à partir de la vallée du Nil, la vague d'islamisation pouvait s'étendre vers l'ouest à travers le Kordofan et le Darfour pour faire, sur les bords du lac Tchad, la jonction avec des populations islamisées déjà par une autre voie, des siècles auparavant.

On peut retenir de ce drame nubien qu'une Eglise pleinement africaine a résisté à I'islam tant qu'elle a pu assurer son ressourcement spirituel, On a l'impression qu'un drame analogue se joue actuellement encore dans la vallée du Nil, au Sud-Soudan, à quelques centaines de kilomètres au sud de l'ancienne Nubie.

\subsection{La voie maritime le long de la côte orientale}

On peut y distinguer nettement deux parties auxquelles correspondent deux phases dans le contact avec l'islam:

- la côte africaine de la Mer Rouge jusqu'à la Corne de l'Afrique,

- la côte africaine de l' Océan Indien.

\subsubsection{L'islam dans les régions de la Mer Rouge et de la Corne de l'Afrique}

La Mer Rouge qui sépare la péninsule arabique du continent africain, n'a jamais constitué une vraie frontière. Bien avant la naissance de Mohammed, l'Ethiopie et les royaumes d'Arabie du Sud essayaient, avec un succès changeant, d'imposer leur 
domination les uns aux autres. La tra- dition musulmane la plus ancienne nous rapporte qu'aux moments les plus critiques pour Mohammed et sa jeune communauté de La Mecque, un bon nombre de disciples furent recueillis par le Négus d'Ethiopie et traités avec bienveillance.

Ainsi n'était-il pas surprenant que, très tôt, une communau- té musulmane s'établit dans le port de Massawa qui fait face à l'Arabie, et dans les îles environnantes. Jusqu'au XIe siècle, d'autres communautés suivirent dans les ports tout le long de la côte occidentale de la Mer Rou- ge. Pendant longtemps ces têtes de pont musulmans, vivant du commerce, reconnurent l'autorité du roi chrétien d'Ethiopie. Lorsqu'à parti r du XIe siècle la domination éthiopienne s'étendit davantage vers le sud, l'islam connut aussi une période d'expansion pacifique parmi les Somalis et les autres populations de la Corne de l'Afrique, grâce aux liaisons commerciales.

C'est en voulant obliger les musulmans à payer tribut, au XVe siècle, que l'Ethiopie provoqua une vive réaction qui, assez vite, prit, du côté musulman, la forme de guerre sainte. Aidés par des armes à feu fournies par les turcs ottomans, les musulmans mirent en danger l'existence même de l'Ethiopie chrétienne. Elle ne fut sauvé que par l'intervention du fameux corps expéditionnaire portugais. Peu à peu elle réussit à éloigner la pression musulmane du plateau éthiopien et cela pratiquement jusqu'à nos jours (cf 6.2.2).

\subsubsection{Les implantations musulmanes sur la côte orientale de l'Afrique}

Lorsque les auteurs arabes parlent de la côte orientale de l'Afrique, ils emploient deux noms différents: la côte des Zanj allant approximativement de Mogadiscio à Tanga, dans le nord de la Tanzanie actuelle, et ensuite «le pays de Sofala» à partir de Zanzibar jusqu'au Mozambique actuel. En fait, cette distinction n'a qu'un intérêt théorique, car la manière d'implantation de l'islam est bien la même du nord au sud.

Les premiers contacts d' Arabes musulmans avec la côte des Zanj devaient remonter sans doute au début de l'islam, mais sûrement au VIIIe siècle et étaient le fait, notamment, d' Arabes yéménites zaydites et donc schismatiques. Aujourd'hui encore, une bonne partie de la population yéménite fait partie de la Shi'a, le parti de 'Ali qui s'était séparé de la gran- de communauté sunnite en 660. Ce sont eux qui fondèrent Mogadiscio en 860, un siècle plus tard Kilwa et sa rivale Mombasa plus au sud, puis Pate, Malindi, Pemba et Zanzibar. Les îles Comores furent visitées par eux dèsle Xe siècle. Dès cette époque, des Perses et des Indiens participèrent à l'implantation de comptoirs musulmans le long de la côte, dans les îles et, plus tard, jusque sur la côte nord-ouest de Madagascar. Au Xe siècle, un géographe musulman décrivit Sofala comme «le point extrême de l' Afrique».

Peu à peu ces comptoirs et colonies musulmanes devenaient des sultanats indépendants; indépendants par rapport à l'empire musulman de Bagdad et de ceux qui lui ont succédé, mais aussi indépendants les uns par rapport aux autres. Tout orientés vers le commerce et bien organisés en cités musulmanes, il ne semble pas qu'il y ait eu de conflits majeurs entre eux. 


\subsubsection{Islamisation et commerce des esclaves}

Contrairement à ce qui s'est passé ailJeurs en Afrique, l'islam est donc resté sur la côte orientale simplement sous forme d'enclaves. Nulle part et à aucun moment, ces sultanats ne cherchaient à se fédérer pour former un ensemble musulman plus grand. Nulle part non plus, ils ne cherchaient à étendre leur influence sur les populations bantoues de l'intérieur en essayant de convertir leurs rois ou leurs chefs. Le seul fruit de cette longue cohabitation entre musulmans arabes et animistes bantous fut la langue swahili, langue à structure bantoue avec un vocabulaire emprunté en grande partie à l'arabe.

L'islamisation de certains centres à l'intérieur du continent fut bien plus tardive et directement liée au commerce de l'ivoire et des esclaves. A partir du XVIIIe siècle, ce commerce florissant donna à Zanzibar une position de leadership sur toute la région. On sait qu'en 1852, il y avait une colonie de musulmans à la cour du Kabaka, le roi des Baganda. A la même époque Ujiji, à la pointe nord du lac Tanganyika, était une bas importante de la traite et donc un centre musulman. L'influence de Zanzibar s'étendait jusqu'au Maniéma dans l'est du Zaàre actuel y constituant une importante enclave musulmane. Sans l'arrivée dés colonisateurs européens n'y aurait-il pas eu tout de même un grand ensemble musulman dans l'est del' Afrique?

\subsection{La voie des caravanes à travers le Sahara}

Après la conquête de l'Egypte, les Arabes poursuivirent immédiatement leur marche victorieuse vers l'ouest, le long de la côte méditerranéenne, à travers toute l'Afrique du Nord. La seule véritable résistance qu'ils rencontrèrent, fut celle des tribus berbères surtout en Numidie.

A cet endroit, il est permis de se demander pourquoi l'Afrique du Nord a si faiblement réagi à l'avancée des troupes musulmanes comparée à la résistance farouche que leur opposèrent les royaumes chrétiens de Nubie pendant des siècles? Une bonne partie de l'Afrique du Nord avait été sous le joug très dur de Bysance, ce qui poussait les chrétiens à accueillir les Arabes en liberateurs. Plus profondément, la vie chrétienne en Afrique du Nord était resté le fait d'une élite, de ceux qui parlaient latin. De plus, au moment de l'arrivée des Arabes, la chrétienté d'Afrique du Nord ne faisait qu'émerger péniblement de quatre siècles de lutes intestines entre adeptes de plusieurs hérésies et défenseurs de la vraie foi(p.ex.la lute de St. Augustin contre donatistes et pélagiens). L'invasion des Vandales ariens avait encore ajouté aux dissensions et aux troubles. Le manque d'unité et d'enracinement de la foi fut déterminant pour le succes rapide de l'avan- cée musulmane. Malgré cela, l'Afrique du Nord n'est pas devenue musul- mane d'un seul coup. Au XIIe siècle il y existait encore des communautés chrétiennes avec une hiérarchie bien établie.

\subsubsection{L'arrivée d'un islam schismatique au sud du Sahara}

Devant la résistance berbère, les Arabes fondèrent la ville de Kairouan qui, de campement militaire comme son nom l'indique, devint, par la suite, la place forte de l'orthodoxie musulmane, un des grands centres intellectuels du monde musulman rayonnant sur toute l'Afrique du Nord et, plus tard, bien au-delà. En effet, si les 
Berbères furent progressivement, et non sans difficultés, soumis à la domination musulmane, ils adoptèrent d'abord la foi musulmane sous une forme schismatique. Du VIIIe au XIe siècle la majeure partie de Maghreb, surtout les zones proches du désert, professaient le Khârijisme, modèle dissident, plus égalitaire, quant à la façon de diriger la communauté musulmane, et correspondant ainsi davantage à l'esprit égalitaire et indépendant des Berbères (cf 5.1). Ce n'est qu'au XIe siècle que le raid guerrier des Almoravides, eux-mêmes fondés par un pré- dicateur kairouannais transplanté sur les bords du Sénégal, fit prévaloir l'orthopraxie sunnite. Les invasions hillaliennes, nouvelle vague de tribus arabes venant d'Arabie au XIIe siècle, finirent par réduire le Khârijisme de manière définitive. Il s'est maintenu jusqu'à nos jours dans quelques îlots du Sahara central (les 'Ibâdites du Mzab) et dans le sud tunisien. Mais il est clairement établi que l'islam traversa le Sahara jusqu'au bord du Niger d'abord sous la forme dissidente du Khârijisme.

Le Sahara, bien moins hostile au Xe siècle qu'il ne l'est aujourdhui, était déjà le domaine des Touareg, branche méridionale de la grande famille berbère. Si euxmêmes étaient plutôt guerriers que commerçants, ils assuraient pourtant la sécurité des routes caravanières et servaient de guides aux commerçants d'Afrique du Nord attirés par la richesse fabuleuse en or des régions au-delà du grand désert. Ils connaissaient notamment le Ghana, royaume puissant, situé dans le nord-ouest du Mali et le sud-est de la Mauritanie actuels.

C'est par ces routes des caravanes que l'islam pénétra au sud du Sahara. Dans la capitale du Ghana, selon des auteurs arabes postérieurs, il y avait, dès le Xe siècle, un quartier musulman situé à l'écart de la ville africaine. Commerçants et lettrés musulmans se rendaient chaque matin au marché ou à la cour du roi, dans la ville païenne, pour traiter leurs affaires. Bien vite, certains de ces lettrés devinrent des conseillers du souverain.

La poussée almoravide, partie des tribus berbères habitant plus à l'ouest, mit fin au royaume du Ghana et aussi à ce premier type de présence musulmane: cohabitation à distance entre musulmans blancs et «païens» africains. Mais le même type de cohabitation pouvait être observé ailleurs: à la cour du Mali naissant, à Gao et, plus à l'est, sur les bords du lac Tchad.

La destruction du Ghana par les Almoravides eut comme autre conséquence l'adoption de l'islam par deux peuples africains: les Toucouleur sur le Moyen-Sénégal et les Sarakolé ou Soninké plus à l'est. Ces derniers étaient, et sont toujours, un peuple extrêmement mobile et commerçant. Bien vite ils prirent le relais des commerçants blancs d'Afrique du Nord. Sous diverses appelations -Marka, Jula, Wangara, etc ... -, ils portèrent, avec leurs marchandises, l'islam à travers le sahel et la savane et jusqu'aux confins de la forêt tropicale. Encore aujourd'hui on peut reconstituer ces anciennes routes commerciales interafricaines en reliant entre eux des villages ou de petits centres de population marka, traditionnellement musulmans, disséminés dans d'autres ethnies.

\subsubsection{Les grands ensembles musulmans du Moyen Age}

Dès le XIIe siècle, le Mali prit la succession du Ghana, groupant dans un vaste empire une multitude de peuples et contrôlant l'extraction et le commerce de l'or. A la différence du souverain du Ghana, l'empereur du Mali se disait musulman. L'islam 
devint la religion de la cour mais avec bien des compromissions païennes selon les circonstances. Au XVe et au XVIe siècle, sous l'empire songhay de Gao, le qualificatif de «religion des princes» fut encore plus justifié. Ce fut l'âge d'or de l'islam ouestafricain.

Les villes universitaires, Tomboutou et Djamena, rélayées plus tard par les cites "hausa" tells que Kano et Zria, furent célébres dans tout le monde musulman en tant que hauts-lieux du savoir islamique. La reputation de certains "ulamâ", savants éssciences islamiques, sétandit jusqu'en Afreique que du Nord et en Orient. Et le paradoxe de l' histoire fut que musulmans, un corps expérditionnaires marocaine, mirent brutalement fin en 1591, à cette grande époque de l'isslam africain.

Sur les bords du lac Tchad, l'islam était arrivé de Libye dès le Xe siècle. Une dynastie musulmane, avec moins d'éclat que celle du Mali et de Gao, put se maintenir au pouvoir pratiquement jusqu'à l'arrivée des troupes coloniales vers 1900 .

Durant cinq siècles la majeure partie de l'Afrique de l'Ouest, entre le désert et la forêt tropicale, fut donc sous une hégémonie musulmane. A part le raid des Almoravides, il ne fut jamais question de guerre sainte. L'islam s'est imposé aux Africains comme la religion du succès et du prestige, possédant en outre un moyen de communication universel: la langue et l'écriture arabes. C'est la religion musulmane qui rendit possible la cohabitation pacifique de nombreux peuples dans un seul ensemble en donnant à ces grands empires leur cohésion. Pourtant, pour toute cette période, il faut parler d'une islamisation superficielle et des élites seule-ment. Les masses rurales, du moment qu'elles s'acquittaient des impôts, ne furent guère concernées par cette religion des chefs. Ceci explique pourquoi, après la disparition du pouvoir central de Gao, pendant deux siècles (XVIIe et XVIIIe), l'islam disparut presque totalement de la scène ouest-africaine. Etait-ce simple coïncidence, qu'à la même époque, le commerce transsaharien fut supplanté, suite à la découverte du pourtour de l'Afrique, par le commerce maritime des puissances européennes?

\subsubsection{Confréries et guerre sainte}

L'ardeur islamique n'était pourtant pas complètement éteinte. Le grand désert renfermait des foyers d'où un réveil islamique pouvait partir. Si la première expansion musulmane en Afrique de l'Ouest était due sur- tout aux réseaux des commerçants, à un pouvoir central unificateur et au prestige de la langue arabe, cultivé dans les centres de sciences religieuses, les porteurs du réveil islamique du XVIIIe etXIXe siècle furent essentiel-

lement les confréries musulmanes.

De quoi s'agit-il? Dès le début, l'islam a connu en son sein des chercheurs de l' Absolu, des maîtres spirituels pour lesquels l'observation des seuls cinq piliers de 1 "islam et l'étude du texte sacré du Coran n'étaient que la base d'unequête plus totale du DieuUnique, quête à laquelle ils consacrèrent toute une vie. Leurs prières et exercices spirituels, tout com- me leurs paroles et leurs écrits, furent bien souvent suspectés par les gar- diens de l'othodoxie, mais le courant mystique (soufisme) ne fut jamais totalement mis au ban de la communauté musulmane.

A partir du XIIe siècle, des écoles ou mieux des ordres mystiques se formèrent, suivant le charisme spirituel d'un fondateur et imitant ses pra- tiques, analogues aux 
ordres religieux ou congrégations en christianisme. Les membres s'appellèrent entre eux «frères» d'où le nom de «confréries».

Le reflux des musulmans d'Espagne à la fin du XVe siècle suite à la reconquista, provoqua une réislamisation et un développement prodigieux de la piété populaire en Afrique du Nord. La principale manifestation en fut une vénération accrue du Prophète de l'islam et des saints personnages locaux. Leurs tombeaux devinrent vite des foyers sprituels (zawia), des centres de rayonnement des confréries. Dès avant la chute de Gao (en 1591), de tels foyers confrériques sont attestés pour le Sahara central et chez les tribus arabo-berbères au nord-ouest de Tombouctou.

Ce ne fut pourtant que deux siècles plus tard que les confréries con- nurent un grand développement au sud du Sahara, notamment dans les dif- férents peuplements fulb 'e (peuls), et furent à l'origine de plusieurs Etats musulmans basés sur les seuls principes de la loi coranique.

La grande dispersion des Fulb 'e (peuls) sur toute l'Afrique de l'Ouest reste un mystère pour les historiens de l'Afrique. Elle fut pratiquement achevée au XVIIIe siècle. Dans quatre foyers peuls, à des milliers de kilomètres de distance, se produisit alors, en l'espace de quelques années (entre 1750 et 1810), une vraie révolution islamique: Des lettrés musulmans formés dans les centres confrériques, se soulevèrent contre des chefs païens ou mauvais musulmans pour mettre en place une théocratie islami- que. Un même processus de renversement des pouvoirs s'opéra au Fouta Djallon dans la Guinée actuelle, au Fouta Toro dans le Sénégal central, au NordNigéria sous la conduite du grand lettré 'Uthmân dan Fodio (1752-1817) et finalement au Maasina dans le centre du Mali actuel.

Ce fut la guerre sainte, mais une guerre sainte qui, sauf dans le cas de 'Uthmân dan Fodio, ne déborda guère sur les peuples voisins. Elle visa Seulement l'islamisation profonde du peuple peul par l'instauration d'un ordre islamique. Mais I "idée de guerre sai nte sous inspiration confrérique était lancée. Elle fut poussée jusqu'au bout par alHajj 'Umar Tall (1797-1864), cinquante ans plus tard. Au nom d'une confrérie rivale, il se tailla, par les armes, un grand empire dans l'ouest du Mali actuel soumettant, dans une guerre crue $11 \mathrm{e}$, indistinctement populations animistes et d'autres déjà islamisées.

\subsubsection{L'islam sous la colonization}

Vers 1900 les troupes coloniales, françaises ou anglaises, trouvèrent ainsi dans le Sahel et la savanne ouest-africaines quelques noyaux forte- ment islamisés et des zones flottantes d'islamisation récente et forcée, à côté de larges étendues encore animistes avec, comme seule présence isla- mique, quelques commerçants musulmans. Même si la conquête coloniale fut sûrement ressentie par certains musulmans africains comme une défai- te de l'islam et donc comme une fitna, une situation intolérable pour un vrai musulman, elle ne rencontra guère de résistance au nom de l'islam, sauf dans les tous débuts au Sénégal. Al-Hajj 'Umar Tall, après un premier affrontement avec les troupes françaises, évita celles-ci en orientant son jihâd vers l'est. Lui-même, ses fils et Samori Touré (1830-1900), plus au sud, n'hésitèrent pas à se servir du colonisateur anglais, présent en Sierra Leone, pour se ravitailler en armes à feu.

On ne peut pas dire non plus qu'il y eut chez les puissances coloniales, la France aussi bien que la Grande Bretagne, une politique bien arrê- tée pour ou contre l'islam en Afrique. Le fait historique est pourtant là qui prouve que durant les quelques 
soixante ans qu'a duré la main-mise européenne sur l'Afrique, l'islamisation a progressé comme jamais auparavant.

Avec leur principe de l'indirect rule, les Britanniques laissèrent le plus possible les choses en place telles qu'ils les avaient trouvées, pourvu que les impôts rentrent et qu'uncertain effort de scolarisation et d'amélioration des conditions de vie soit fournis. La conséquence du système del' indirect rule fut que les chefs et émirs musulmans, surtout au Nord-Nigéria, purent, tranquillement et en toute légalité, mener une politique d'islamisation de larges couches de populations animistes en les pressurant pour l'impôt et en déversant sur elles seules tout le poids des exigences du colonisateur. Devenir musulman fut alors le seul moyen de s'en tirer à bon compte.

L'administration directe des Français demande un examen plus nuancé: certains administrateurs favorisèrent ouvertement l'islam, soit par anti-cléricalisme, très à la mode au début du siècle, soit par conviction sincère que l'islam pourrait constituer une étape intermédiaire sur la route qui conduirait les «indigènes» vers la civilisation et le progrès. Générale- ment les administrateurs trouvèrent parmi les musulmans les auxiliaires ouverts et instruits dont ils avaient besoin pour «pacifier» le pays. Les chefs et personnalités musulmanes furent souvent plus connus et plus accessibles que les chefs traditionnels. Ainsi l'islam gagna vite en prestige aux yeux de la majorité animiste en fournissant au colonisateur ses colla- borateurs indispensables. Ces derniers, à leur tour, profitèrent largement de leur situation pour la propagation de la religion musulmane en milieu encore animiste.

D'autre part, la nouvelle sécurité des routes, le développement des moyens de transport et des voies de communication permirent aux musulmans, notamment aux commerçants et religieux de tout rang, une mobili- té nouvelle pour étendre le prosélytisme musulman dans les campagnes. Les centres administratifs, les écoles, le service militaire obligatoire et les prestations de travail forcé, tout ce qui comportait départ et déracinement du terroir coutumier ou brassage de populations différentes, fut en même temps lieu et occasion d'islamisation, superficielle certes, mais effective.

Dans tout cela, l'administration coloniale surveilla étroitement les chefs religieux musulmans. Un fichier central fut établi à cet effet à Dakar, siège du Gouverneur Général. La visée principale de cette surveillance fut de tenir l'islam africain le plus éloigné possible de l'islam arabe. Les allers et venues entre l'Orient et l'Afrique de l'Ouest furent contrôlés avec précision.

Les représentants officiels de l'islam comme les dignitaires dés gran- des confréries, s'adaptèrent vite à la nouvelle situation et surent en tirer une augmentation de leur prestige si ce n'est de leurs richesses. Les seuls heurts majeurs entre la colonisation française et les musulmans eurent lieu autour de la naissance des deux confréries purement africaines: le Mouri- disme au Sénégal et la Hamâlliyya au Soudan (Mali actuel) (cf 3.5.8 et 3.5.4). Les deux confréries furent, à leurs débuts, accusées de xénophobie, d'instigatrices à la révolte contre le pouvoir colonial et rejetées comme hérétiques par les autres musulmans. Plustard, l'administration coloniale appuya encore de la même manière la majorité des musulmans tradition- nels contre les premiers réformateurs formés dans les universités islamiques du Caire et d' Arabie, lorsque ceux-ci s'attaquèrent aux abus de l'islam confrérique.

Tout au long de la période coloniale, le colonisateur chercha donc à «domestiquer» l'islam et à l'utiliser pour sa politique. L'islam, de son côté, fit bon ménage avec les «mécréants» et en profita fortement pour se faire connaître, se faire 
bien voir et finalement s'imposer dans des zones qui lui avaient été fermées jusqu'alors. On peut tout simplement s'étonner qu'il n'eût pas progressé davantage.

\subsection{L'islam et les indépendances}

Un autre sujet d'étonnement pourrait être l'absence totale de motivation islamique dans la lutte des différents pays pour l'indépendance. Les indépendances furent acquises, pour la plupart, en dehors de toute référence à l'islam ou au désir d'instaurer un ordre islamique, autre preuve, s'il en faut, du degré de «domestication» subie par l'islam sous la colonisation. Dans les mobilisations des partis politiques pour l'indépendance et encore pendant une bonne dizaine d'années après, les leaders, pourtant majoritairement des musulmans, ne firent jamais appel à une argumentation allant dans le sens du jihâd islamique ou de l'instauration d'un ordre islamique sur un des modèles du passé. Le fait que l'on ne puisse plus dire la même chose de certains leaders africains d'aujourd'hui, montre bien la transfor- mation en cours de l'islam africain.

\subsection{Les fadeurs de l'expansion musulmane au sud du Sahara}

Après ce long parcours géographique et historique, il suffit de répondre en quelques lignes à la question: $\mathrm{Qu}$ 'est-ce qui, en définitive, a [ait adopter l'islam à plus de cent millions d'Africains de préférence à toute autre religion? Les situations et motivations sont diverses, certes, mais il y a quelques constantes qui se dégagent comme valables pour l'cnscmhlc du continent.

\subsection{Islam et commerce des esclaves}

La guerre sainte ne fut certernement pas le facteur le plus détenninant. Elle n'a joué un role que localement. On peut s'en réjouir, L'islamisation en Afrique subsuhuricnne n'a pas été, en premier lieu, une histoire d'afrrontcments violents laissant dans la mémoire des peuples des traces dou-loureuses et des antagonismes latents, toujours prêts à resurgir, comme c'est le cas dans d'autres régions du monde touchées par l'islam.

Il existe pourtant un de ces souvenirs douloureux, lié à la présence de l'islam en Afrique, mais pas uniquement à elle. Depuis l'apparition des premiers commerçants sur les bords du Niger ou sur la côte orientale de l'Afrique, la présence du musulman, surtout s'il est de race blanche, est liée instinctivement au souvenir de l'esclavage et du commerce des esclaves.

L'esclavage, sous des formes diverses, a existé en Afrique bien avant l'islam. Mais à bien des endroits, avec plus ou moins d'intensité selon les époques, les commerçants musulmans ont fait des esclaves, tout naturelle- ment, une des marchandises privilégiées du commerce transsaharien ou maritime. La recherche des esclaves est devenue pour ainsi dire une des principales raisons de leur venue et de leur présence en Afrique Noire. Ce souvenir douloureux, avec sa forte charge affective d'opposition raciale, est loin d'être effacé. De tout temps, il a constitué un des obstacles durables à une islamisation massive. Aujourd'hui encore, il est un obstacle psychologique majeur qui empêche les musulmans africains, blancs et noirs, de s'unir sous 
là seule bannière de l'islam. La ligne de démarcation entre les deux, qui passe au sud du grand désert, reste une zone permanente de conflits, même si pratiquement tous, de part et d'autre, se réclament de la même foi.

\subsubsection{La réussite dans le commerce}

Parmi les facteurs qui ont favorisé l'adoption de l'islam par les Africains, il faut nommer en premier lieu le commerce. Historiquement il est certainement premier. Plus profondément, vu les origins de l'islam, il y existe un lien intrinsèque entre la mise en valeur rapide des biens temporels et le culte du Dieu Unique; entre la réussite matérielle et la soumission au Tout-Puissant. La première est le signe evident que la seconde est bien agréée. De plus, comme le suggère le Coran lui-même, les richesses temporelles, signe de bénédiction divine, peuvent et doivent servir à (faire pencher les coeurs de ceux qui sont indécis), vers l'islam. Il est impossible d'évaluer combien ce prosélytisme par les biens matériels et les moyens financiers a joué dans lislnmisation tout au long de l'histoire. Le fait qu'il est encore couramment pratiqué aujourd'hui.

Un autre aspect du lien étroit entre commerce et islam a eu son importance en Afrique dans le processus dislamisation: L'islam est une religion au dogme clair. au culte simple niais d'une expressivité vigoureuse. Il peut être pratiqué nimporte où et n'est lié à aucun sacerdoce ni à aucune fonction spéciale ni à aucun lieu particulier. Dans sa pratique laplus fruste, il cadre ainsi parfaitement avec la mobilité soit du commerçant, soit du nomade. Dans sa régularité contraignante pour l'accomplissement des préceptes religieux, notamment durant le Ramadan, comme aussi dans ses ex igcnces communautaires, 1 "islam fait pourtant appel à un genre de vie de type citadin et indépendant. Seul le commerçant aisé peut organiser sa vie de manière à correspondreà toutes les exigences du culte et à toutes les prescriptions sur le plan social. Une comparaison plus approfondie entre lislam, «religion de partout», et la religion traditionnelle fortement liée à un terroir précis et limité, serait très éclairant pour voir combien la conversion à l'islam ouvre à l'homme africain des horizons nouveaux. Le commerçant, itinérant ou installé au milieu d'une population animiste, constitue une tentation permanente, une invitation constante à s'échapper vers un univers spirituel et matériel plus vaste et autre.

\subsubsection{Le prestige du savoir}

L'islam a pénétré en Afrique chez des peuples à tradition purement orale et sans écriture, alors que la vie du musultnan ne peut se concevoir sans lien avec le Livre, le texte arabe du message coranique. Savoir lire et écrire l'arabe devient le moyen d'accéder à un univers spirituel nouveau. Durant toute une période, les sciences religieuses musulmanes ont atteint un grand développement et rayonnement en Afrique. Même si elles n'ont jamais eu une grande originalité, ce qui compte aux yeux des gens simples, ce qui impressionne et qui fait peur parfois, c'est Je prestige de l'écriture réservé à quelques uns. Ils s'en servent et parfois l'exploitent.

D'autre part, les lettrés en arabe ont été très tôt les instruments indis- pensables pour l'administration centrale et la cohésion des grands empires. Souvent les princes, eux-mêmes guère lettrés, s'entouraient de savants musulmans comme conseillers, secrétaires, ambassadeurs ... Malgré les heurts qu'il ait pu y avoir ici ou fa, entre lettrés 
et pouvoir politique, par exemple au X Ve siècle entre Sonni Ali Ber (1464-1492) de Gao et les 'ulamâ' de Tombouctou, en général les sciences religieuses étaient intimement liées à l'exercice du pouvoir. Accéder, par la conversion à l'islam, au savoir religieux, signifiait en même temps s'approcher de ceux qui déte- naient le pouvoir.

Ceci devenait encore plus évident avec le développement des réseaux confrériques. Chaque lieu d'implantation confrérique autour d'un personnage «charismatique» ou d'un de ses lieutenants était à la fois pôle de rayonnement du savoir religieux et de l'exercice d'un pouvoir attaché à ce savoir. Les grands leaders qui appelaient à la guerre sainte au XVIIIe et XIXe siècle étaient en même temps de grands lettrés, porteurs de la force spirituelle du texte sacré et du glaive de la guerre sainte qui n'était que le prolongement de la force émanant du savoir religieux.

Même sous la colonisation, pourtant laïque et parfois anti-cléricale, ce

«bi-norne», savoir et pouvoir, continuait à fonctionner au profit de l'islam à travers tous ceux quel' administration coloniale recrutait parmi les lettrés musulmans: interprètes, secrétaires, grades inférieurs de l'armée ...

\subsubsection{Le bouleversement dû à la modernité}

Pourtant, tous les facteurs analysés sommairement jusqu'ici, n'ont eu, jusqu'au XIXe siècle, qu'un impact très partiel sur la plupart des populations sédentaires et agriculteurs des savanes africaines. Avec la sécurité retrouvée des voies de communication et le développement de moyens de transport nouveaux, ces facteurs traditionnels augmentent leur impact et leur attractivité au milieu des populations animistes. Mais, en définitive, c'est le choc même de la rencontre entre l'univers socio-religieux traditionnel et la modernité apportée par la colonisation qui a ouvert les milieux animistes à l'adoption de l'islam.

La religion traditionnelle, fruit de longs siècles d'expérience humaine ritualisée, constitue un cadre de vie globale pour un milieu de vie et d'en- vironnement bien précis. La modernité, dans le sillage de la colonisation, oblige de plus en plus d' Africains à sortir de leur milieu de vie, de leur terroir ancestral. Où trouver une nouvelle sécurité, un nouveau cadre de vie familiale, sociale, spirituelle, alors que l'on sait que, hors de chez soi, l'ancien cadreest inopérant? La communauté musulmane, au contact de laquelle on a vécu depuis longtemps, s'offre tout naturellement, et pratiquernent sans exiger de rupture avec le passé, pour donner à l'homme africain, déraciné, un nouveau cadre de vie aussi global que l'ancien, mais qui est rnaiutenant illimité dans l'espace.

Le développement des centres administratifs et commerciaux oû 1'islam était toujours présent et prépondérant, plus tard l'exode rural et l'urbanisation rapide, comportant déracinement et brassage depopulations, sont les facteurs décisifs de l'islamisation en cours en Afrique depuis près d'un siècle 


\section{References}

Abdulaziz, Mohamed H. (1993). History of the Spread of Islam on the East African Coast. Islam in Africa South of the Sahara. Konrad-Adenauer-Stiftung: Sankt Augustin, 31-46. [in English].

Brenner, L. (1987;1988). Sufism in Africa in the Seventeenth and Eighteenth Century. Islam et Sociétés au sud du Sahara, No. 1 (1987), 106-108; No. 2 (1988), 80-93. [in English].

Cissoko, S. M. (1969). Traits fondamentaux des sociétés du Soudan occidental duXVIIe au début du X/Xe siècle. BIFAN t. XXXIII, 1-30. [in French].

Cissoko, S. M. (1975). Tombouctou et l'Empire songhay. Dakar-Abidjan: Nouvelles Editions Africaines. [in French].

Constantin, F. (éd.) (1987). Les voies de l'islam en Afrique Orientale. Paris: Karthala. [in French].

Coulon, C. (1983). Les musulmans et le pouvoir en Afrique Noire. Paris : Karthala. [in French].

Cuoq, J. (1975). Recueil des sources arabes concernant l'Afrique occidentale du Villeau XVIe siècle. Paris: C.N.R.S. [in French].

Cuoq, J. (1984). Histoire de l'islamisation de l'Afrique de l'Ouest des origines à [afin du XVIe siècle. Paris: Geuthner. [in French].

Gagnon, M. (1990). Islam in the Horn of Africa: Penetration and Expansion. Encounter No. 163, Rome: PISAI. [in English].

Guennec-coppens, Françoise Le, Caplan, I Pat (éds). (1991). Les Swahili entre Afrique et Arabie. Nairobi-Paris: Credu/ Karthala. [in French].

Renault, F., Daget, S. (1988). Les traites négrières en Afrique. Paris: Karthala. [in French].

Robinson, D. (1988). La guerre sainte d'al-Hajj Umar. Paris: Karthala. [in French].

Sanankoua, B. (1990). Un empire peul au XIXe siècle. Paris: Karthala-ACCT. [in French]. Zeltner, Jean-Claude. (1980). Pages d'histoire du Kanem pays tchadien. Paris: L'Harmattan. [in French]. 